\title{
Experimental Qualification of New Instrumentation for Lead-Lithium Eutectic in IELLLO Facility
}

\author{
Alessandro Venturini ${ }^{\mathrm{a}}$, Francesca Papa ${ }^{\mathrm{b}}$, Marco Utili $^{\mathrm{c}}$, Nicola Forgione ${ }^{\mathrm{a}}$ \\ ${ }^{a}$ University of Pisa, Dipartimento di Ingegneria Civile e Industriale, L.go L. Lazzarino 2, 56122 Pisa, Italy \\ ${ }^{b}$ DIAEE - Nuclear Section, "Sapienza" University of Rome, Corso Vittorio Emanuele II, 244, 00186 Rome, Italy \\ ${ }^{c}$ ENEA Brasimone, 40032 Camugnano, Bologna, Italy
}

The experimental facility IELLLO was installed in ENEA Brasimone R.C. in 2007, aiming to support the design of liquid Test Blanket Modules that will be installed in ITER and to contribute to the development of Lead-Lithium Eutectic (LLE) technologies. IELLLO has been recently upgraded by installing instrumentation relevant for ITER application. Differential pressure transducers, a Coriolis and a thermal mass flow meters were installed in the facility. An experimental campaign was planned, setting two objectives. The first objective was to qualify the instrumentation for flowing LLE The installation of a differential pressure transducer across each flow meter made also possible to characterize the pressure drops across these instruments. The second objective of this activity was to improve the results of the 2015 campaign by analyzing the performances of the main components of the loop at lower mass flow rates (namely $0.5-1.2 \mathrm{~kg} / \mathrm{s}$ ) and by quantifying the pressure drops across the main components. The investigated flow rates were chosen to be relevant for the LLE loop of the WCLL TBS (Water Cooled Lead-Lithium Test Blanket System). This work presents the results of the experimental campaign, paying particular attention to underline the lessons learned on how to correctly operate instrumentation for LLE.

\section{Keywords: Lead-Lithium Eutectic, Instrumentation, Pressure Transducer, Flow Meter, WCLL TBS}

\section{Introduction}

Lead-Lithium Eutectic (LLE) is the breeder candidate of three of the four Breeding Blanket (BB) concepts that are currently under investigation in Europe as "driver" and "advanced" blankets for DEMO [1]

Instrumentation is essential for the operation of the LLE loops of any BB concept which uses LLE as a breeder. As commercial instrumentation is not currently produced for LLE, R\&D activities are required in order to select and adapt instruments able to work in flowing LLE, allowing to monitor the required parameters (temperature, pressure, mass flow rate, level, tritium concentration, impurities, etc.).

IELLLO (Integrated European Lead Lithium LOop) facility was used in the past to characterize the performances of a Vortex flow meter and of absolute pressure transducers [2]. Then, the absolute pressure transducers were tested at higher pressures in the experimental campaigns that aimed to simulate the consequences of an In-box LOCA for the HCLL TBS (Helium Cooled Lithium-Lead Test Blanket System) [3]. A guided-microwave level meter was also successfully tested in these campaigns.

The experimental campaign described in this paper has been primarily conceived in support to the design activities of the EU WCLL TBS (Water Cooled LithiumLead). WCLL is a candidate to become the "driver" blanket at the gate review in 2024. This campaign had three objectives:

- to characterize Coriolis and thermal flow meters (TFM);
- to test differential pressure transducers;

- to use these instruments to analyze the performances of the loop (pressure drops, characteristics of the pump, efficiency of the economizer).

\section{Description of the facility}

IELLLO (Fig. 1) is an eight-shaped loop in which LLE circulates at a maximum mass flow rate of about $2.5 \mathrm{~kg} / \mathrm{s}$ and can reach a maximum temperature of $550^{\circ} \mathrm{C}$ in the hot side, while the cold side is always kept below $350^{\circ} \mathrm{C}$ to protect the pump and the Coriolis flow meter. With respect to the loop described in [5], IELLLO underwent a few modifications in 2015 and in 2018. In 2015 , the original mechanical pump was substituted with a permanent magnet pump, while the new instruments to be tested were added in 2018 .

LLE is circulated by the permanent magnet pump, located above the storage tank S01, and increases its temperature going through the economizer E01, a counter-current pipe in pipe regenerative heat exchanger. Then, the alloy can pass through the $40 \mathrm{~kW}$ electrical heater $\mathrm{S} 05$ or it can maintain its temperature constant by means of a bypass line. Regardless of the path chosen for the operation, LLE passes through the test section and it cools down in the economizer and in the air cooler E02, before returning to the permanent magnet pump.

The two new flow meters (FT-03 and FT-04 in Fig. 1) have been installed in series downstream of the bypass valve EV-RP01. When the bypass valve is kept closed, the two flow meters are in series also with the Vortex (FT-01). The five differential pressure 
transducers (DPT01-DPT05) have been installed across the pump, the hot side of the economizer, the air cooler and the two new flow meters. Due to a limit working temperature of $400^{\circ} \mathrm{C}$, the remote connections of these instruments have to be installed on two dedicated branches, connected to the loop by a Tee. A dedicated heating band has to be installed on each branch.

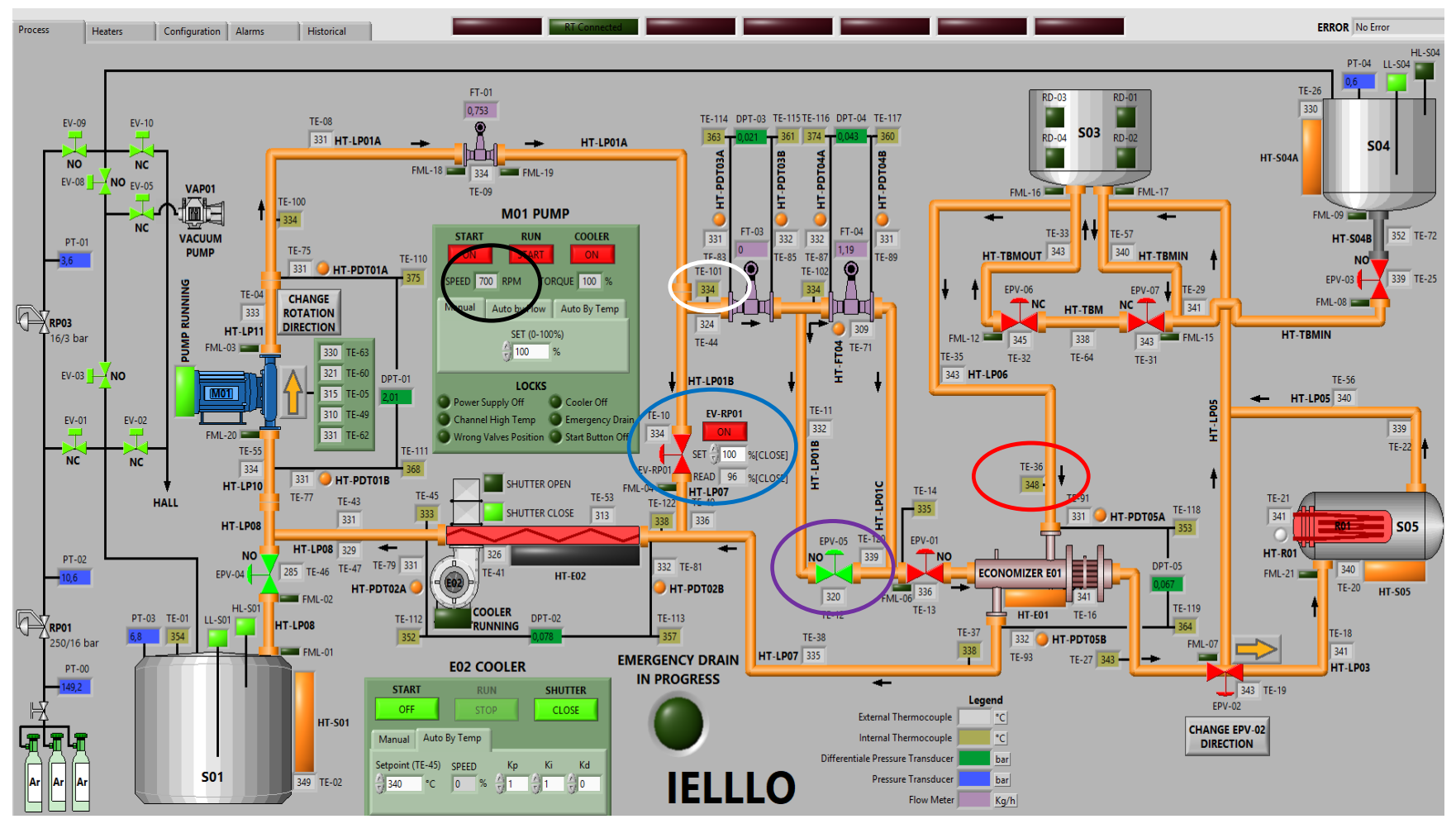

Fig. 1. Sketch of IELLLO facility taken from the Data Acquisition and Control System. The five colored circles underlines the most important parameters for this campaign: the speed of the pump (black), the temperature at the inlet of the thermal flow meter (white), percentage of closure of the bypass valve (blue), the position of the valve EPV-05 (purple) and the maximum temperature of the loop (red).

\section{Test matrix and preliminary operations}

The parametric space (Table 1) for this experimental campaign was composed by 192 points. Each test lasted 30 minutes in order to reduce the impact of statistical oscillations on the results. At the end of each test, the average values were calculated (all the signals were acquired with a frequency of $1 \mathrm{~Hz}$ ). After each test, the parameters were changed and the steady state was reached before starting the next acquisition.

Table 1. Parametric space of the experimental campaign.

\begin{tabular}{ccc}
\hline $\begin{array}{c}\text { Pump speed } \\
{[\mathrm{rpm}]}\end{array}$ & $\begin{array}{c}\text { Closure of bypass } \\
\text { valve }[\%]\end{array}$ & $\begin{array}{c}\text { Max. T of the loop } \\
{\left[{ }^{\circ} \mathrm{C}\right]}\end{array}$ \\
\hline 50 & 0 & 330 \\
100 & 20 & 400 \\
200 & 40 & 450 \\
300 & 60 & 500 \\
400 & 80 & \\
500 & 100 & \\
600 & & \\
700 & & \\
\hline
\end{tabular}

The experimental part of the activity began after the facility was degassed with alternated argon flushes and vacuum, in order to prevent LLE oxidation. First, the instruments were calibrated by means of a Genii Druck multipurpose calibrator. Then, the error in the signal chain was estimated. To do this, the instruments were disconnected and a known current was applied to the signal chain: the relative error between the value read by the Data Acquisition and Control System and value corresponding to the imposed current constitutes the error of the signal chain.

In order to perform a preliminary assessment of the differential pressure transducers, the hydrostatic $\Delta \mathrm{P}$ was used as a comparison: as the flanges of the remote connections of 3 transducers are installed at two different heights, a $\Delta \mathrm{P}$ was measured when LLE was loaded and left in stagnant conditions. The difference between the measured and the theoretical values (i.e., calculated from the $\Delta \mathrm{z}$ between the two connections) was negligible, highlighting the good operation of the instruments.

Fig. 1 shows IELLLO during the preparation of the experiments, with five colored circles that underlines the most important parameters to be checked during the experiments. In particular, it was essential that the temperature at the inlet of the thermal flow meter (white circle) was constantly kept at about $330^{\circ} \mathrm{C}$ to avoid issues to the Coriolis mass flow meter (located immediately after the thermal flow meter and indicated 
as FT-04). Moreover, the valve EPV-05 (purple circle) was always kept closed to drive all the flow through the Coriolis mass flow meter.

\section{Uncertainty analysis}

An important part of the experimental campaign is to evaluate the measuring system error, as defined in the paper by Moffat [6]. In this experimental campaign, it was decided to consider the errors related to:

- the accuracy of the instrument (taken from the manufacturer's specifications);

- the error of the signal chain;

- the oscillations of the value measured by the instrument due to its precision.

This procedure has been performed on every instrument and for each test. Table 2 shows the initial data for each instrument for this analysis.

Table 2. Accuracy and error in the signal chain for each instrument.

\begin{tabular}{llll}
\hline Instr. & Accuracy & $\begin{array}{l}\text { Full } \\
\text { Output (FSO) }\end{array}$ & $\begin{array}{l}\text { Error in the } \\
\text { signal chain }\end{array}$ \\
\hline FT-01 & $0.5 \% \mathrm{read}$. & $0.943 \mathrm{~m} 3 / \mathrm{h}$ & 0 \\
FT-03 & $0.1 \mathrm{~kg} / \mathrm{s}$ & $5 \mathrm{~kg} / \mathrm{s}$ & 0 \\
FT-04 & $0.2 \%$ FSO & $4 \mathrm{~kg} / \mathrm{s}$ & $0.001 \mathrm{~kg} / \mathrm{s}$ \\
DPT1 & $0.1 \%$ FSO & $5 \mathrm{bar}$ & $0.001 \mathrm{bar}$ \\
DPT2 & $0.1 \%$ FSO & $0.7 \mathrm{bar}$ & 0 \\
DPT3 & $0.1 \%$ FSO & $1.1 \mathrm{bar}$ & 0 \\
DPT4 & $0.1 \%$ FSO & $1.4 \mathrm{bar}$ & 0 \\
DPT5 & $0.1 \%$ FSO & $0.8 \mathrm{bar}$ & 0 \\
\hline
\end{tabular}

After having evaluated the standard deviation $S$ of each measured signal, the total measuring system error was calculated following the error propagation theory:

$$
\varepsilon=\sqrt{\varepsilon_{a c c}^{2}+\varepsilon_{c h}^{2}+S^{2}}
$$

where $\varepsilon_{c h}$ is the error of the signal chain, $\varepsilon_{a c c}$ is the error related to the accuracy of the instrument and $S$ is:

$$
S=\sqrt{\frac{1}{N-1} \sum_{i=1}^{N}\left|x_{i}-\bar{x}_{\text {exp }}\right|^{2}}
$$

where $x_{i}$ is the measured value, $\bar{x}_{\text {exp }}$ is their mean and $N$ is the number of data collected during one test.

Table 3 shows the total measuring error for each instrument, expressed as a percentage averaged on the 48 tests at each maximum temperature of the loop. The percentage error of the Vortex flow meter is very high, but it has to be mentioned that this instrument displayed a 0 every time that the flow rate was higher than its Full Scale Output $\left(0.943 \mathrm{~m}^{3} / \mathrm{h}\right)$. This behaviour leads to high standard deviation in some tests, which in turn leads to high errors. When averaging on the group of 48 tests, this causes the total average error to be high too.
Table 3. Average total measuring error for each instrument.

\begin{tabular}{lllll}
\hline Instrument & $330^{\circ} \mathrm{C}$ & $400^{\circ} \mathrm{C}$ & $450^{\circ} \mathrm{C}$ & $500^{\circ} \mathrm{C}$ \\
\hline FT-01 [\%] & 6.114 & 8.543 & 7.437 & 11.779 \\
FT-03 [\%] & 3.111 & 2.386 & 2.281 & 2.174 \\
FT-04 [\%] & 0.211 & 0.242 & 0.207 & 0.208 \\
DPT1 [\%] & 0.122 & 0.127 & 0.127 & 0.124 \\
DPT2 [\%] & 0.107 & 0.114 & 0.116 & 0.115 \\
DPT3 [\%] & 0.101 & 0.101 & 0.101 & 0.101 \\
DPT4 [\%] & 0.100 & 0.101 & 0.100 & 0.101 \\
DPT5 [\%] & 0.017 & 0.017 & 0.017 & 0.017 \\
\hline
\end{tabular}

The measuring error of thermocouples is separately discussed, as their accuracy varies with temperature and an additional source of error has to be taken into account: an error associated to the calibration instrument (GE DPI 620 Genii) has to be considered. Type K thermocouples have a declared accuracy of

$$
\begin{aligned}
& \text { - } \quad \pm 1,5^{\circ} \mathrm{C} \text { from }-40{ }^{\circ} \mathrm{C} \mathrm{a}+375^{\circ} \mathrm{C} \text {; } \\
& \text { - } \quad \pm 0.004 \cdot \mathrm{T}\left[{ }^{\circ} \mathrm{C}\right] \text { above }+375^{\circ} \mathrm{C} \text {. }
\end{aligned}
$$

When the accuracy is added to the error in the calibration $\left( \pm 0.3^{\circ} \mathrm{C}\right)$ and to the error in the signal chain (varying from -0.5 to $+0.8^{\circ} \mathrm{C}$ ) with the root-sum-square method, the total error lies between 1.53 and $2.08^{\circ} \mathrm{C}$ in all the cases of this experimental campaign. The rootsum-square of this error and of the standard deviation (of each thermocouple independently) constitutes the total measuring error of the thermocouple, whose values range from $0.22 \%$ to $0.37 \%$.

The propagation of the error from the primary quantities (e.g., temperature) to the derived quantities (e.g., efficiency of the economizer) has been calculated by means of the method of sequential perturbations, proposed by Moffat [6].

LLE properties are involved in the calculation of some derived quantity and, thus, the error associated with each property has also to be considered in the propagation. LLE density has been used in the conversion of the flow rate measured by the Vortex flow meter from $\mathrm{m}^{3} / \mathrm{h}$ to $\mathrm{kg} / \mathrm{s}$. The error associated to LLE density has been considered $0.3 \%$ [7]. After having propagating the error, the average percentage error for the converted mass flow rate is about $0.32 \%$ of the average of the converted values. Instead, the specific heat has been used in the evaluation of the power exchanged in the economizer. The error associated to LLE specific heat has been considered 3\% [8]. The two selected correlations are the ones that are also suggested in [9].

Table 4. Percentage propagated errors.

\begin{tabular}{llll}
\hline Error in & $400^{\circ} \mathrm{C}$ & $450^{\circ} \mathrm{C}$ & $500^{\circ} \mathrm{C}$ \\
\hline Efficiency [\%] & 7.46 & 4.01 & 2.60 \\
Power [\%] & 13.13 & 11.48 & 11.03 \\
\hline
\end{tabular}


Table 4 shows the propagated error expressed as an average percentage for the efficiency of the economizer and for the power exchanged in the economizer.

\section{Experimental results}

The experiments can be divided into three groups:

- $\quad$ flow meters comparison;

- components and instruments pressure drops;

- $\quad$ efficiency and power of the economizer.

\subsection{Flow meters comparison}

Before to start the experimental characterization of the pressure drops, the performances of the flow meters were assessed in order to select the most reliable one. A first set of tests was performed when the bypass valve was completely closed, so that all the flow meters were in series. Fig. 2 shows the results of this comparison. The error bars for the Vortex and the Coriolis are included, but their values are too low to be distinguished. Table 5 shows the average differences between the three instruments. The percentage differences are almost constant for the entire pump speed range, ruling out the possibility of a malfunctioning instrument.

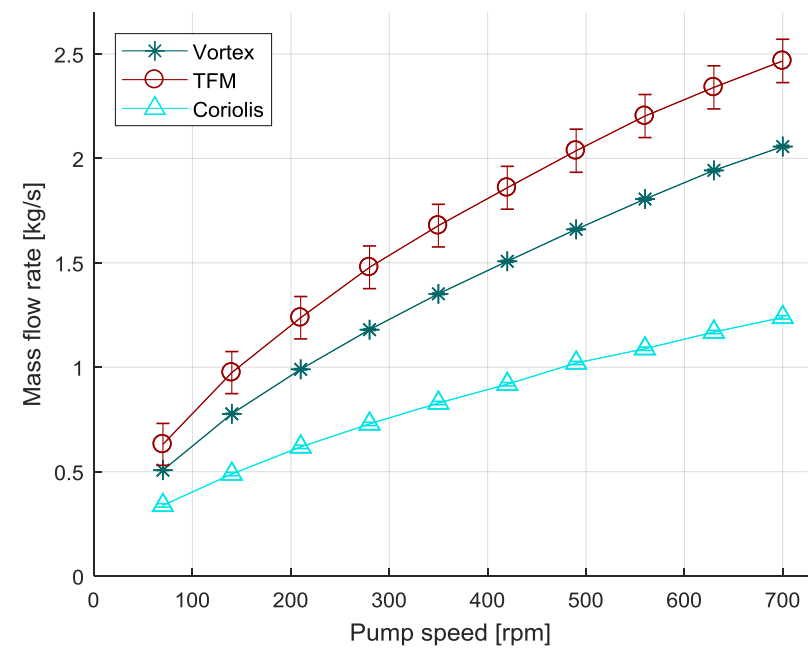

Fig. 2. Behavior of the three flow meters varying the speed of the pump (with the bypass valve closed).

The Coriolis and the TFM could also be compared at different closures of the bypass valve. The results of these tests are shown in Fig. 3 and Fig. 4 and high The data of the thermal flow meter obtained at the lowest pump speeds (50 rpm and, to a lesser extent, $100 \mathrm{rpm}$ ) are not fully reliable, as it was working too close to its lower working limit (about $0.5 \mathrm{~kg} / \mathrm{s}$ ).

After an extended discussion with the suppliers, it was concluded that the thermal flow meter has to be considered the most reliable, thus being used as a reference in the following tests, as:
- the principle of operation of the TFM is the simplest one, thus unpredicted phenomena that can hinder its operation can be excluded;

- the Coriolis should measure the density, but it did not: a discussion with the supplier led to the possible conclusion that LLE density is out of the measuring range (i.e. $10,000 \mathrm{~kg} / \mathrm{m}^{3}$ );

- the Vortex flow meter is not completely reliable as LLE density and viscosity might affect the correct formation of eddies.

Table 5. Average difference between the flow meters.

\begin{tabular}{lll}
\hline \multirow{2}{*}{ Pump speed } & $\begin{array}{l}\text { TFM-Vortex } \\
{[\%]}\end{array}$ & $\begin{array}{l}\text { TFM-Coriolis } \\
{[\%]}\end{array}$ \\
\hline $100 \%$ & 18.13 & 66.29 \\
$70 \%$ & 20.37 & 66.50 \\
$40 \%$ & 22.47 & 67.96 \\
$10 \%$ & 21.75 & 60.27 \\
\hline
\end{tabular}

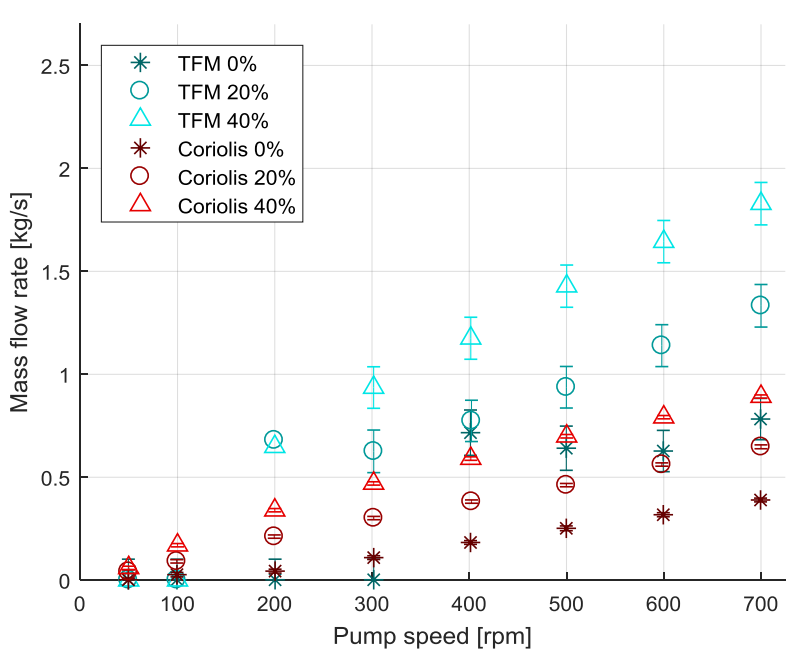

Fig. 3. Flow meter comparison with the bypass closed at $0 \%, 20 \%$ and $40 \%$.

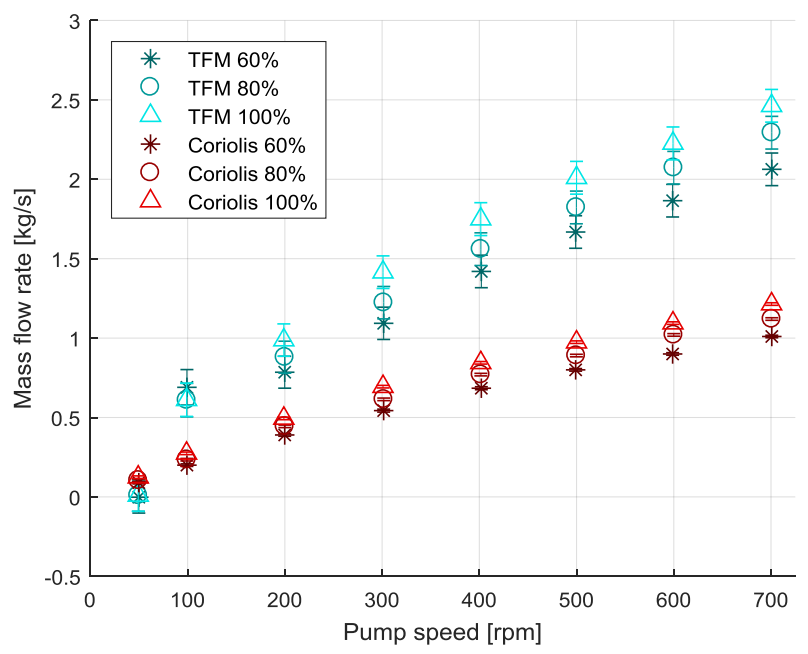

Fig. 4. Flow meter comparison with the bypass closed at $60 \%, 80 \%$ and $100 \%$.

\subsection{Components pressure drops}

In the following figures the component pressure drops are shown. The differences of pressure shown 


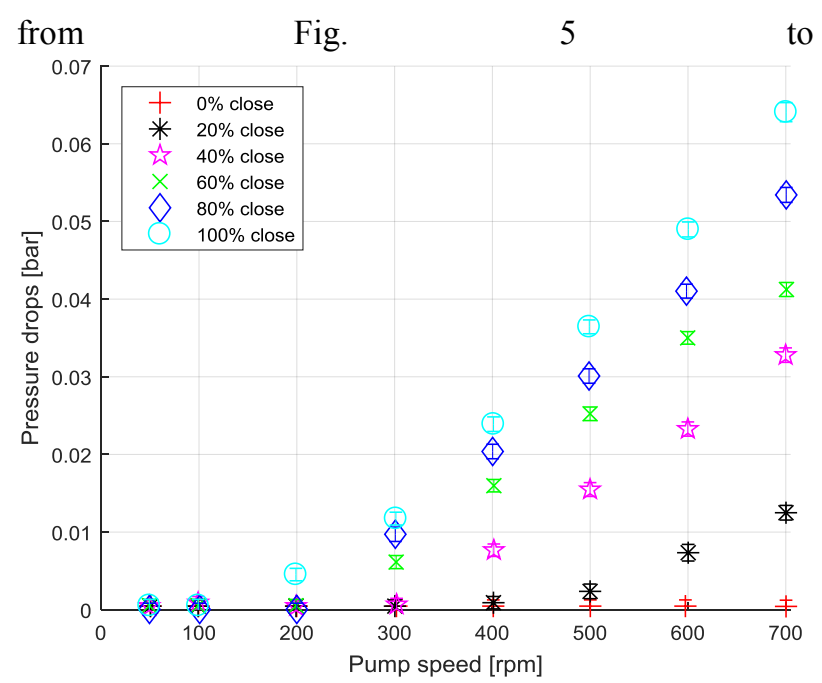

Fig. 7 highlight that the tested differential pressure transducers can successfully be used in LLE, paying particular attention to their installation. Given their low working temperature of $400^{\circ} \mathrm{C}$ and the high melting point of LLE $\left(235^{\circ} \mathrm{C}\right.$, if exactly eutectic [10]), the working range is not big. Moreover, the need of a dedicated branch to install the two remote connection, which was filled by stagnant LLE, makes the correct operation of these instruments not easy. The installation of a dedicated heating band, and of a thermocouple inside the branch (and in contact with LLE) to control it, is fundamental to avoid freezing or overheating.

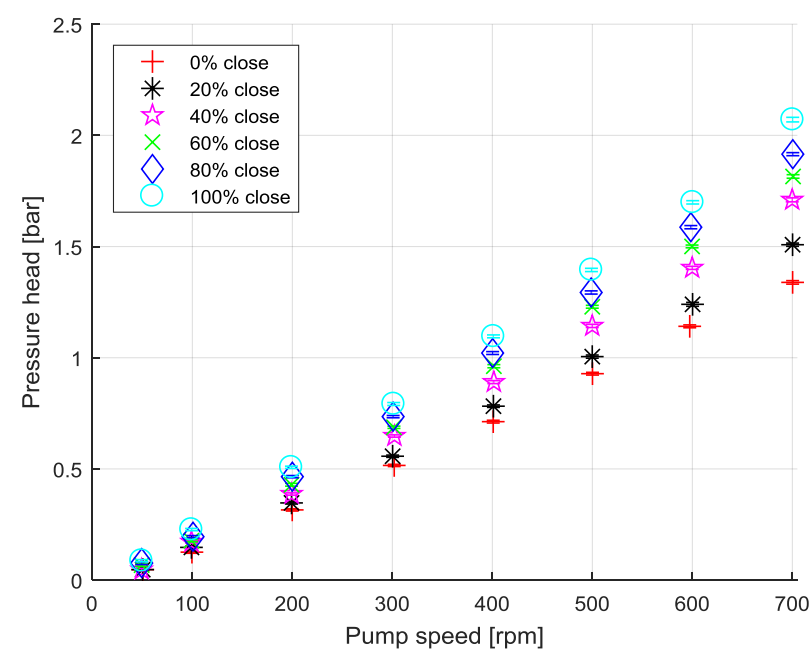

Fig. 5. Characteristic curve of the system measured by DPT01 at different closures of the bypass valve.

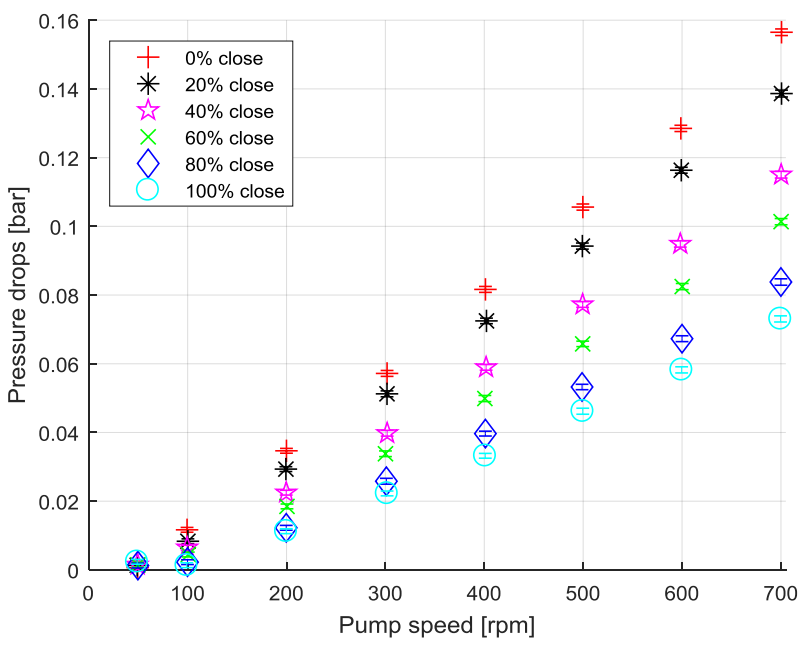

Fig. 6. Pressure drops across the air cooler measured by DPT02 at different closures of the bypass valve.

Studying the pressure drops across the components of IELLLO is relevant for the WCLL TBS, as similar technologies will be likely used for the LLE loops. The pressure drops across the air cooler (Fig. 6) have a big impact on the total pressure drops of the loop (Fig. 5), especially when the bypass is more open (they represent about the $11 \%$ of the total when the bypass is fully open). Instead, the pressure drops across the economizer (Fig. 7) are less significant, with a maximum impact of about the $3 \%$ of the total pressure drops.

The differential pressure transducers installed across the thermal and Coriolis mass flow meters show that their pressure drops are almost negligible, with slightly higher values for the TFM (Fig. 8 and Fig. 9). Therefore, the use of these instruments in the LLE loops would not represent an issue for the pumping system.

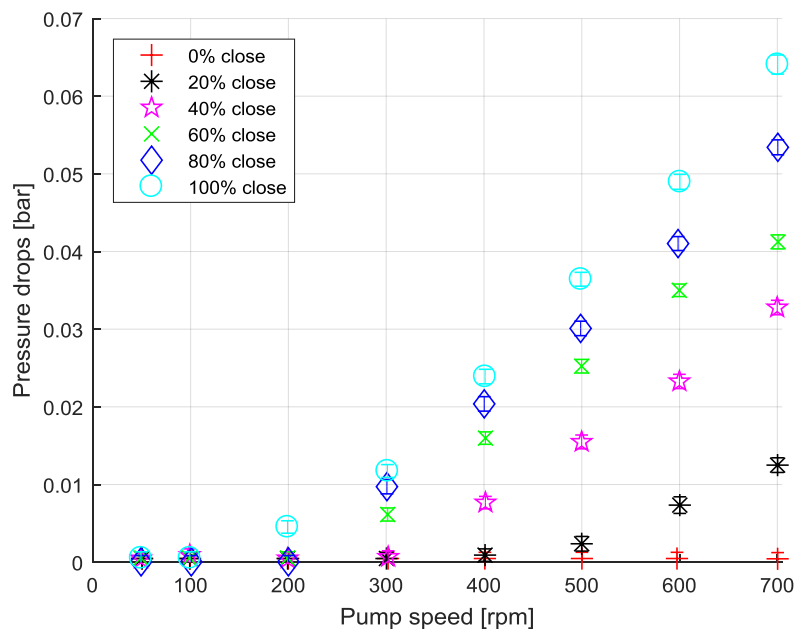

Fig. 7. Pressure drops across the economizer measured by DPT05 at different closures of the bypass valve. 


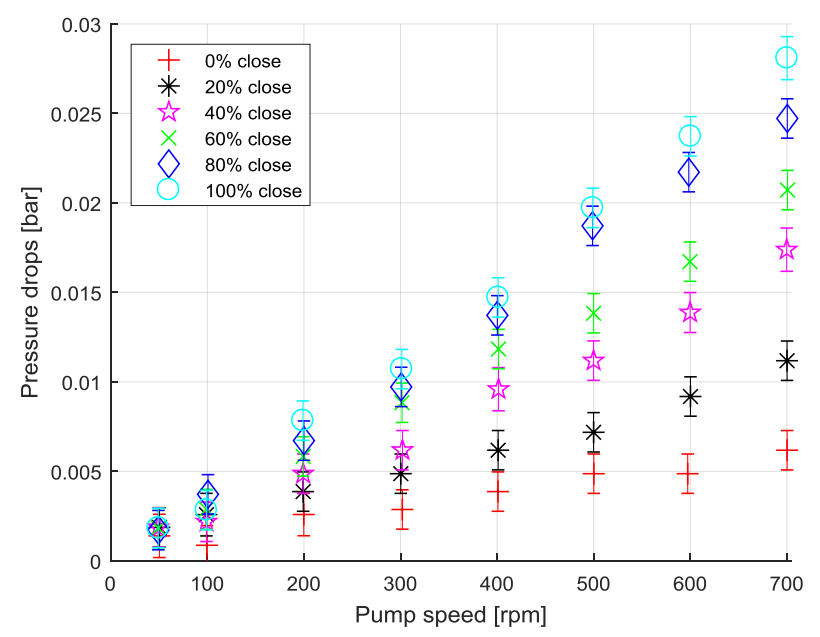

Fig. 8. Pressure drops across the TFM measured by DPT03 at different closures of the bypass valve.

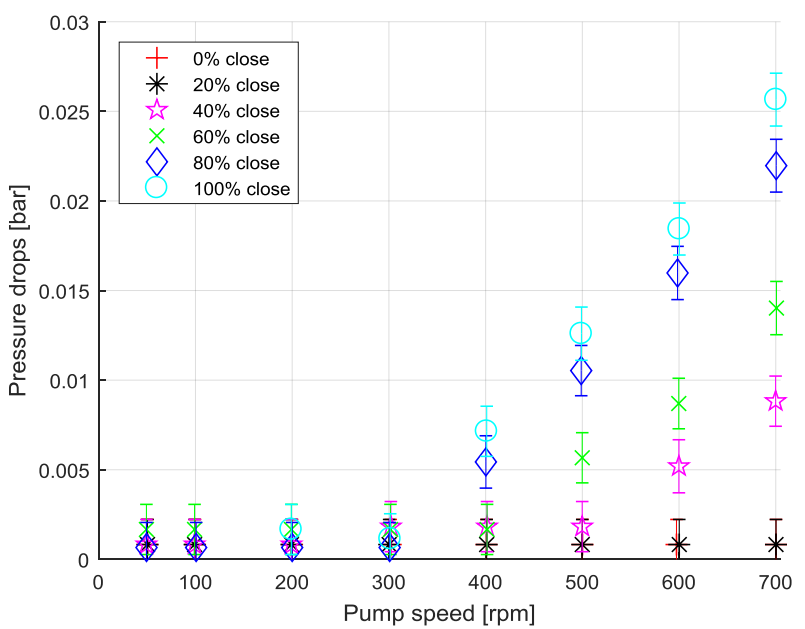

Fig. 9. Pressure drops across the Coriolis measured by DPT04 at different closures of the bypass valve.

\subsection{Performances of the economizer}

Fig. 10 and Fig. 11 show the efficiency of the economizer at two maximum temperatures of the loop, while Fig. 12 shows the maximum powers exchanged. The error bars have been removed by the efficiency plots to not compromise their readability.

The decreasing trend of the efficiency at higher flow rate was already highlighted by the experimental campaign of 2015 [2], but the use of the thermal flow meter allowed to reach lower flow rates, which are more relevant towards the design of the WCLL TBS and ancillary systems. Nevertheless, some perturbations occurred at small flow rates (below $1 \mathrm{~kg} / \mathrm{s}$ ) for two reasons: the lower working limit of TFM caused some spurious measurements; the power given to LLE by the thermal flow meter influenced the temperature at the inlet of the cold side of the economizer.

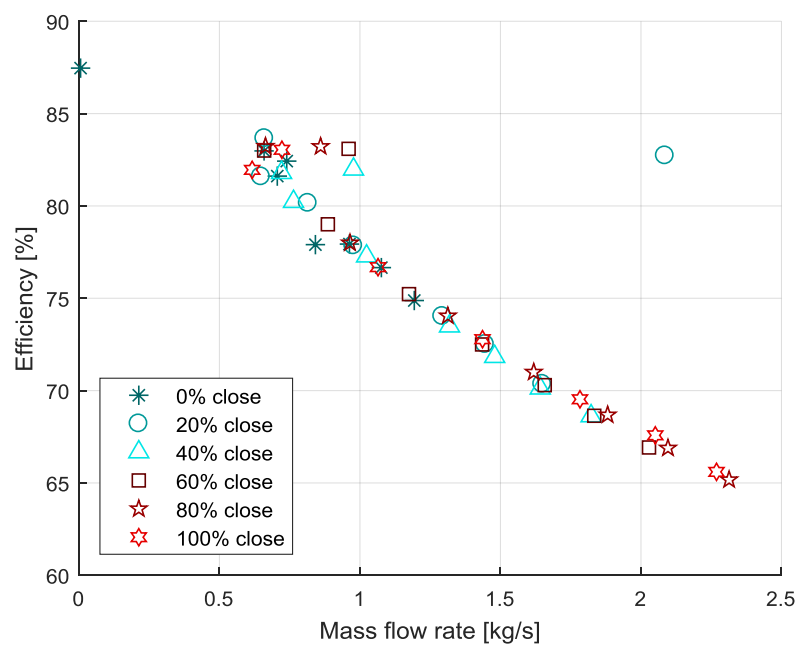

Fig. 10. Efficiency of the economizer when the maximum temperature of the loop is $400^{\circ} \mathrm{C}$.

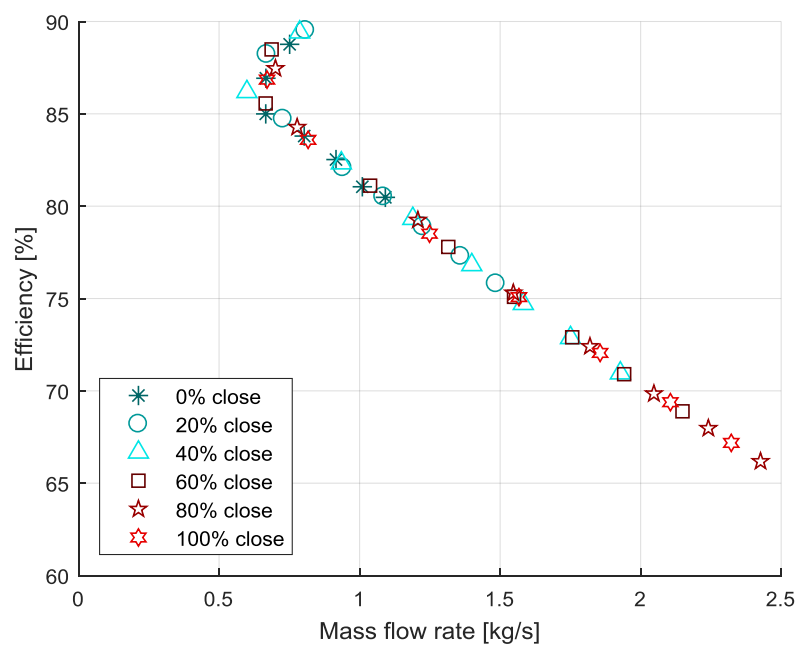

Fig. 11. Efficiency of when the maximum temperature of the loop is $500^{\circ} \mathrm{C}$.

The power exchanged (Fig. 12) increases with the mass flow rate: given that the specific heat is constant, the power trend is determined only by the relative change rate of the mass flow rate and of the temperature difference. In these tests, the change rate of the mass flow rate was bigger than that of the temperature difference, causing the power to increase at higher flow rates. 


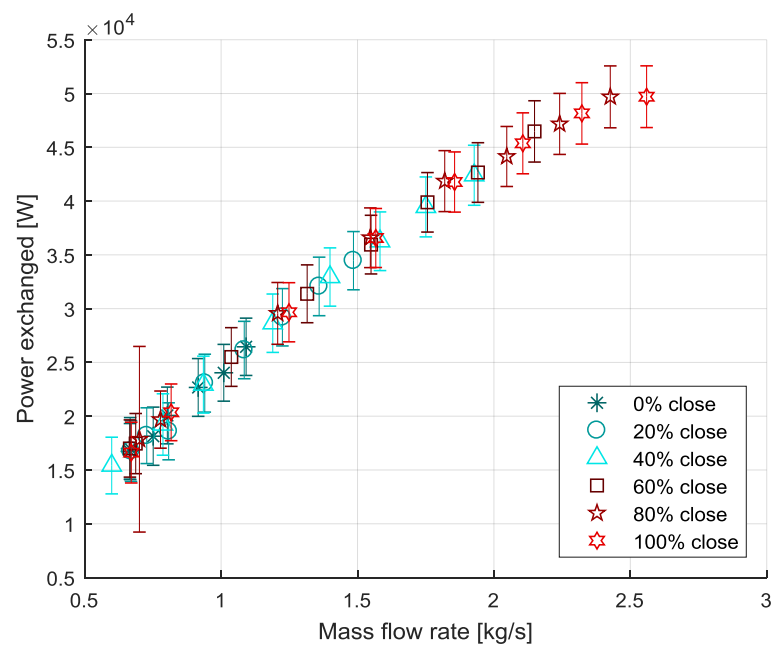

Fig. 12. Power exchanged in the economizer when the maximum temperature of the loop is $500^{\circ} \mathrm{C}$.

\section{Conclusions}

Two new mass flow meters were tested: firstly they were compared with the Vortex flow meter, with the bypass valve completely closed so that the three instruments were in series; then they were compared at different closures of the bypass valve. The thermal flow meter was considered the most reliable of the three flow meters. As a drawback, this design of the thermal flow meter has a lower working limit of $0.5 \mathrm{~kg} / \mathrm{s}$.

The new flow meters allowed to evaluate the pressure drops and the performances of the economizer and of the air cooler at smaller flow rate than those used in [2], when only the Vortex was installed in IELLLO. The flow rates below $1 \mathrm{~kg} / \mathrm{s}$ are most relevant towards the design of the HCLL or WCLL TBS. The economizer proved to be a very efficient component with a maximum efficiency of about $87.5 \%$ when the mass flow rate is about $0.5 \mathrm{~kg} / \mathrm{s}$ and the maximum temperature of the loop is $500^{\circ} \mathrm{C}$.

Five differential pressure transducers were installed and tested. They were firstly tested with stagnant $\mathrm{Pb}-$ $15.7 \mathrm{Li}$, checking the hydrostatic pressures and, then, used to measure pressure drops on the main components of the loop. Pressure drops across the thermal flow meter are very small, with a maximum of about 0.03 bar at about $2.5 \mathrm{~kg} / \mathrm{s}$. Coriolis flow meter has even smaller pressure drops (about 0.025 bar at about $2.5 \mathrm{~kg} / \mathrm{s}$ ).

\section{Acknowledgments}

The work leading to this publication has been partially funded by Fusion for Energy under the specific grant FPA-372-SG04. Special thanks go to our F4E colleagues and ENEA colleagues involved in this project.

\section{References}

[1] G. Federici et al., An overview of the EU breeding blanket design strategy as an integral part of the DEMO design effort, Fusion Engineering and Design 141 (2019) 30-42.

[2] A. Venturini et al., Experimental and RELAP5-3D results on IELLLO (Integrated European Lead Lithium LOop) operation, Fusion Engineering and Design 123 (2017) 143-147.

[3] A. Venturini et al., Experimental campaign on pressure wave propagation in LLE, Fusion Engineering and Design 136 (2018) 809-814.

[4] A. Venturini et al., Experimental investigation on HCLLTBS In-box LOCA, Fusion Engineering and Design 146 (2019) 173-177.

[5] M. Utili, et al., The European breeding blanket test facility: an integrated design to test European helium cooled TBMs in view of ITER, Fusion Engineering and Design 84 (2009) 1881-1886.

[6] R. J. Moffat, Describing the Uncertainties in Experimental Results, Experimental Thermal and Fluid Science 1 (1988) 3-17.

[7] S.V. Stankus et al., An Experimental Investigation of the Density and Thermal Expansion of Advanced Materials and Heat-Transfer Agents of Liquid-Metal Systems of Fusion Reactor: Lead-Lithium Eutectic, High Temperature. Vol. 44, No. 6 (2006) 829-837.

[8] B. Schulz, Thermophysical properties of the $\operatorname{Li}(17)$ $\mathrm{Pb}(83)$ alloy, Fusion Engineering and Design 14 (1991) 199-205.

[9] D. Martelli et al., Literature review of lead-lithium thermophysical properties, Fusion Engineering and Design 138 (2019) 183-195.

[10] P. Hubberstey, T. Sample, M.G. Barker, Is Pb-17Li really the eutectic alloy? A redetermination of the lead-rich section of the $\mathrm{Pb}$-Li phase diagram $(0.0<\mathrm{xLi}(\mathrm{at} \%)<22.1)$, Journal of Nuclear Material 191-194 (1992) 283-287. 